\title{
The Use of High Dose Eltrombopag in the Management of Sepsis-Associated Thrombocytopenia in Critically Ill Patients
}

\author{
Md. Jahidul Hasan ${ }^{1 *}$, Raihan Rabbani², Shihan Mahmud Redwanul Huq ${ }^{2}$ \\ 1 Department of Clinical Pharmacy, Square Hospitals Ltd., Dhaka, Bangladesh \\ 2 Internal Medicine and ICU, Square Hospitals Ltd., Dhaka, Bangladesh
}

\begin{abstract}
Introduction: Sepsis is a life-threatening condition, and sepsis-associated thrombocytopenia (SAT) is a common consequence of the disease where platelet count falls drastically within a very short time. Multiple key factors may cause platelet over-activation, destruction and reduction in platelet production during the sepsis. Eltrombopag is a thrombopoietin receptor agonist and is the second-line drug of choice in the treatment of chronic immune thrombocytopenia (ITP).

Aim of the study: The objective of this study was to observe the therapeutic outcome of high dose eltrombopag in SAT management in critically ill patients.

Material and Methods: This 6-month-long single group, observational study was conducted on seventeen ICU patients with SAT. Eltrombopag $100 \mathrm{mg} /$ day in two divided doses was given to each patient. Platelet counts were monitored. A low platelet blood count returning to $150 \mathrm{~K} / \mu \mathrm{L}$ or above, is taken as indicative of a successful reversal of a thrombocytopenia event.

Results: The mean Apache II score of patients $(n=17)$ was 18.71 ( $p$-value: $>0.05)$. No eltrombopag-induced adverse event was observed among the patients during the study period. Thrombocytopenia events were reversed successfully in $64.71 \%$ of patients $(11 ; n=17)$ within eight days of eltrombopag therapy.

Conclusions: The therapeutic potentiality of high dose eltrombopag regime in the management of sepsis-associated thrombocytopenia was found clinically significant in over two-thirds of critically ill adult patients enrolled in the study. These data may point to a new strategy in the management of acute type of thrombocytopenia in septic patients.
\end{abstract}

Keywords: Eltrombopag, sepsis, thrombocytopenia, megakaryocytes, thrombopoietin receptor

Received: 15 May 2019 / Accepted: 05 August 2019

\section{INTRODUCTION}

Sepsis, severe sepsis and septic shock are analogous terms used to clinically characterize disease severity [1]. Sepsis and septic shock are the major contributors to mortality rates of critically ill patients in intensive care units (ICU). Severe sepsis, with or without septic shock, is considered to occur when two or more systemic inflammatory response syndromes (SIRS) ensue within the first twenty-four hours of a patient being admitted to an ICU [2].

American College of Chest Physicians/Society of Critical Care Medicine Consensus Conference (1991) offered new definitions for some terms, and broad definitions of sepsis and the systemic inflammatory response syndrome were proposed, along with detailed physiologic variables by which a patient could be categorized [3].

The Third International Consensus Definitions for Sepsis and Septic Shock (2016) recommended that sepsis associated with organ dysfunctions is assessed by the Sequential Organ Failure Assessment (SOFA) system, and "Septic shock" is defined as the advanced subset of sepsis where abnormalities of circulatory, cellular and metabolic functions are observed [1]. Approximately 750,000 sepsis cases were recorded per year in the United States (US) and caused to 215,000 deaths with an economic loss of 16.7 billion US Dollars [4]. 
Thrombocytopenia is a condition where the platelet level in blood is below $150 \mathrm{~K} / \mu \mathrm{L}$ due to different risk factors [5]. The normal platelet level in the blood is 150$400 \mathrm{~K} / \mu \mathrm{L}$, and generally, the platelet count is higher in females than that of males [6]. Thrombocytopenia is a common phenomenon among septic patients, [5] with a $20 \%-40 \%$ incidence rate in critically ill ICU patients [7] and presents as a significant risk factor [5].

The risk factors for thrombocytopenia in critically ill patients have been the subject of many reported studies [8], with sepsis was identified as the most prevalent risk factor [9-11].

However, sufficient clinical data, contributing to an in-depth understanding of sepsis/severe sepsis/septic shock-associated thrombocytopenia event, remains unavailable, though data does exist concerning sepsis-associated disseminated intravascular coagulation (DIC) and immune thrombocytopenic purpura (ITP) is available $[12,13]$.

Thrombocytopenia of less than $80,000 \mathrm{~K} / \mu \mathrm{L}$ platelets develops in approximately $40 \%$ of patients with sepsis and can fluctuate with the severity of the diseases [14].

The management of ITP and sepsis-associated thrombocytopenia (SAT) is a critical task even for the physicians $[15,16]$.

Eltrombopag is a low molecular weight, synthetic biphenyl hydrazone, nonpeptide circulating thrombopoietin (TPO) receptor agonist (TPO-RA) [16]. Recently, eltrombopag has been approved by the US Food and Drug Administration (FDA) for the treatment of chronic immune thrombocytopenia (ITP) in children patients [17]. Stephan et al. (1999) showed that eltrombopag had positive dose-dependent activities in raising platelet count in adult patients with chronic ITP [8]. Studies found a good response in ITP with a high dose of methylprednisolone, dexamethasone and hydrocortisone $[16,19]$.

There is a minimal number of clinical evidence concerning the use of eltrombopag in SAT management.

The study aimed to evaluate the potentiality of the high dose eltrombopag in the treatment of SAT in ICU critically ill patients.

\section{- METHODS}

The study was conducted for six months, between May 2018 and October 2018. It was a single group, prospective, observational study, undertaken in the ICU of a tertiary level hospital in Dhaka, Bangladesh. During the study period, the usual ICU setups and the medical data obtained from admitted critically ill patients were used. Data were collected daily. This observational study was conducted without modifying the existing treatment protocols of the ICU. [The study was not a clinical trial or a part of any clinical trial. Standard clinical data collected in the ICU was used to assess the clinical outcome measures when a high dose of eltrombopag was prescribed in the management critically ill patients with SAT.

Data relating to patients who had been prescribed eltrombopag were analysed to assess the therapeutic outcome of a 50mg dose of eltrombopag in the management of SAT in critically ill patients.

\section{Inclusion criteria}

- Patients admitted to the ICU within the first seventy-two hours of developing sepsis or septic shock symptoms;

- Patients aged between 45 - 65 years;

- Patients having a blood platelet count less than 50 $\mathrm{K} / \mu \mathrm{L}$;

- Only patients with no history of any steroidal therapy within the last 72 hours of initiating eltrombopag therapy were included.

\section{Exclusion criteria}

- Moderate to severe hepatic impairment as determined the Child-Pugh classification;

- Different types of metabolic disorders including thyroid disorders, acid-base imbalance, glucose metabolism disorders, calcium metabolism disorders and mitochondrial diseases;

- Chronic critical illness within the last thirty days;

- Recent post-surgical patients;

- Current history of alcoholism;

- Patient on any antiplatelet therapy;

- Recent history of any active bleeding or any type of haemorrhage;

- A history of thrombocytopenia within the last 15 days.

\section{Sample size calculation}

The outcome of this study was categorical, and the prevalence was estimated to $5 \%$ points of the true value, with $95 \%$ confidence. Septic patients with sepsis-related thrombocytopenia, admitted to the ICU during the defined period, constituted the study population. 
A sample size 87 (n) was estimated for this study using the formula of single proportion. When the sample exclusion criteria were applied, the final sample size obtained during the allotted study period was reduced to 17 patents.

In keeping with the sample inclusion criteria, during the study period, a total of ninety-six patients with SAT, all of South Asian origin, were initially included in the study. However, when the sample exclusion criteria were applied, only 26 patients complied with both inclusion and exclusion criteria.

During the study period, nine patients were excluded, two patients have quitted the ICU against medical advice and seven patients were prescribed steroids as part of their required treatment and so were excluded from the study. The remaining seventeen patients, aged between 45 - 65 years, had a diagnosis of sepsis with or without accompanying septic shock, confirmed by the medical staff, as was a recently developed thrombocytopenia confirmed to be the consequence of the sepsis-development.

The APACHE II score of each patient was recorded prior to eltrombopag therapy, using the APACHE II Scoring system software incorporated in the hospital's "Hospital Integrated System (HIS)"

Platelet levels were monitored on daily-basis after the commencement of eltrombopag therapy.

\section{Design of eltrombopag therapy}

Eltrombopag tablets (Julphar, Gazipur, Bangladesh) $(100 \mathrm{mg} /$ day, were given orally in two divided doses.

The drug-administration time was fixed at 5 am and $5 \mathrm{pm}$ to accommodate the ingestion of the medicament on an empty stomach. Each dose consisted of a $50 \mathrm{mg}$ tablet, crushed into a slurry, which was administered either directly orally or through a nasogastric tube.

The critical care pharmacist monitored the patients for any eltrombopag related side effects and any other drug-induced adverse reaction during the study period.

A low platelet blood count returning to $150 \mathrm{~K} / \mu \mathrm{L}$ or above, is taken as indicative of a successful reversal of a thrombocytopenia event.

The Square Hospital Ethical Committee granted ethical approval for this study in January 2018.

Each patient signed a written informed consent form before being included in the study.

\section{Statistical analysis}

Data were analysed using IBM SPSS statistics (version 22) software. The results are expressed as Mean (SD) and percentages. Pearson Chi-square test was used for categorical variables. The level of significance was alpha $=0.05$.

\section{RESULTS}

The patients $(n=17)$ of this study were aged between 47-65 years (Mean(SD) 54.62 (11.32).

Ten (10) were female patients and seven (7) were male patients (Table 1).

Before commencing eltrombopag therapy, the mean procalcitonin level was $18.42 \mathrm{ng} / \mathrm{mL}$ (min level $0.7 \mathrm{ng} /$ $\mathrm{mL}$ and max level $75 \mathrm{ng} / \mathrm{mL}$ ), (Table 1). There was no significant correlation between the procalcitonin level and thrombocytopenia event in the patients ( $\mathrm{p}$-value: $0.556)$.

The mean Apache II score was 18.71(5.44), (range: 0-71) (Table 1).

The highest bilirubin level was $2.3 \mathrm{mg} / \mathrm{dL}$ (mean (SD) $1.43(0.51) \mathrm{mg} / \mathrm{dL})$.

Within the first eight days of initiation of eltrombopag therapy, platelet counts returned to normal level

Table 1. Demographic and pathological information

\begin{tabular}{|c|c|c|}
\hline Characteristics & Variables & P-values \\
\hline \multicolumn{3}{|l|}{ Age (year) } \\
\hline Mean & 54.62 & \\
\hline Range (min-max) & $47-65$ & \\
\hline \multicolumn{3}{|l|}{ Gender } \\
\hline Male & 7 & \\
\hline Female & 10 & \\
\hline \multicolumn{3}{|c|}{ Procalcitonin (<0.1 ng/mL) } \\
\hline Mean & 18.43 & \multirow{2}{*}{0.556} \\
\hline Range (min-max) & $0.7-75.0$ & \\
\hline \multicolumn{3}{|c|}{ Apache II Score (0-71) } \\
\hline Mean & 18.71 & \multirow{2}{*}{0.980} \\
\hline Range (min-max) & $10.0-28.0$ & \\
\hline \multicolumn{3}{|c|}{ Bilirubin $(0.2-1.2 \mathrm{mg} / \mathrm{dL})$} \\
\hline Mean & 1.43 & \multirow{2}{*}{0.516} \\
\hline Range (min-max) & $0.6-2.3$ & \\
\hline \multicolumn{3}{|c|}{ Initial Platelet count (150-400 K/ $\mu \mathrm{L})$} \\
\hline Mean & 31.71 & \multirow{2}{*}{0.980} \\
\hline Range (min-max) & $20.0-47.0$ & \\
\hline
\end{tabular}


(150 to $450 \mathrm{~K} / \mu \mathrm{L}$ of blood) in eleven patients $(64.71 \%$; $\mathrm{n}=17$ ) (Figure 1) whereas in other six patients (35.29\%; $\mathrm{n}=17$ ), no response was observed (Figure 1 ).

The platelet count in one patient (patient no. 7) returned to within normal limits on the fifth day of treatment. In the remaining ten patients, SAT was resolved with the platelet count returning to within normal limits, albeit with some fluctuation in level by the end of the study. (Table 2).

During the study period, the critical care pharmacists of the ICU reported that all seventeen patients had no specific interaction profile between other prescribed medicaments and eltrombopag.

\section{Discussion}

$64.71 \%$ of study patients showed a positive response to eltrombopag and complete recovery from thrombocytopenia was gained within eight days of therapy.

The dose of eltrombopag was $100 \mathrm{mg} / \mathrm{day}$, which is higher than the recommended daily dose of $50 \mathrm{mg}$, once daily, for the treatment of ITP. The recommended dose of eltrombopag in East Asian populations, e.g., Japanese, Chinese, Taiwanese and Korean, is $25 \mathrm{mg}$

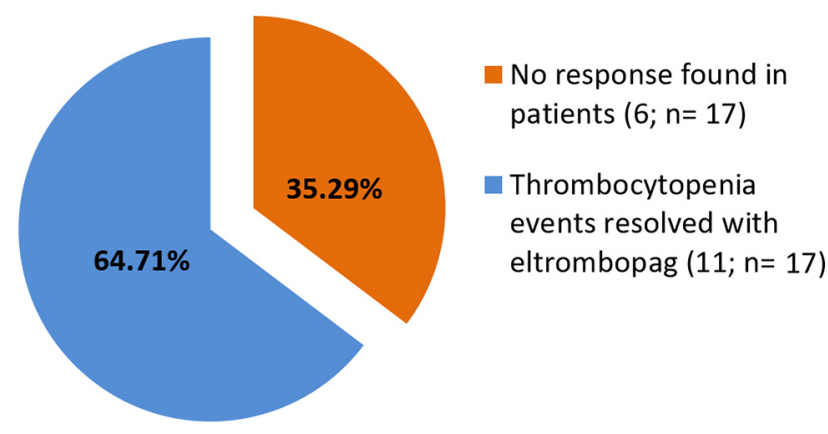

Fig. 1. Outcomes of eltrombopag therapy in sepsis-associated thrombocytopenia once daily [20]. However, previous experience in this ICU, using the recommended dose of eltrombopag in SAT management had not been found to give satisfactory results. The accepted protocol was to administer double the recommended dose. This decision had been taken some time previous to the commencement of the present study, which therefore followed the ICUs recommended higher dose and as has been stated (vide supra) was therefore not an experimental study.

Eltrombopag was developed in 2004, and after getting approval from the US FDA, it was strongly recommended as the second-line treatment option of chronic ITP [21]. ITP is an autoimmune disease characterized by antiplatelet antibody-mediated platelet destruction, reduced platelet count, and that can cause life-threatening severe bleeding $[17,18]$. Antiplatelet antibodies play a pivotal role in developing ITP-associated thrombocytopenia [22]. The primary mechanism behind this outcome is the proliferation and differentiation of megakaryocyte progenitor cells in the bone marrow through the activation of the thrombopoietin receptors and the c-Mpl after binding of eltrombopag with the thrombopoietin receptors [23].

Fragmentation of megakaryocytes, also known as haematopoietic cells, is found in the bone marrow yields platelet, and the process is called 'thrombopoiesis. To accomplish this process, a hormone synthesized by the liver, known as thrombopoietin (TPO) plays a pivotal role in the thrombopoiesis process [24]. TPO has positive role in proliferation and expression of megakaryocytes [25]. Other than TPO, there are some other factors, such as cytokines including interleukin-3 (IL-3), interleukin-6 (IL-6), interleukin-11 (1L-11) and stem cell factor (SCF) have a positive impact on the thrombopoiesis [24]. The half-life of platelet is eight days, and after that course, they pass through the BAK/ BCL-XL interaction system for programmed intrinsic

Table 2. Day and patient wise effect of eltrombopag on platelet count

\begin{tabular}{|c|c|c|c|c|c|c|c|c|c|c|c|}
\hline \multirow[b]{2}{*}{ Day } & \multicolumn{11}{|c|}{ Patient $(n=17)$ wise platelet count } \\
\hline & $\begin{array}{c}\text { Patient } \\
1\end{array}$ & $\begin{array}{c}\text { Patient } \\
2\end{array}$ & $\begin{array}{c}\text { Patient } \\
3\end{array}$ & $\begin{array}{c}\text { Patient } \\
4\end{array}$ & $\begin{array}{c}\text { Patient } \\
5\end{array}$ & $\begin{array}{c}\text { Patient } \\
6\end{array}$ & $\begin{array}{c}\text { Patient } \\
7\end{array}$ & $\begin{array}{c}\text { Patient } \\
8\end{array}$ & $\begin{array}{c}\text { Patient } \\
9\end{array}$ & $\begin{array}{c}\text { Patient } \\
10\end{array}$ & $\begin{array}{c}\text { Patient } \\
11\end{array}$ \\
\hline Day 1 & 24 & 30 & 21 & 37 & 26 & 34 & 39 & 30 & 47 & 20 & 24 \\
\hline Day 2 & 39 & 41 & 32 & 53 & 30 & 69 & 69 & 45 & 65 & 23 & 36 \\
\hline Day 3 & 68 & 70 & 58 & 51 & 65 & 96 & 121 & 61 & 76 & 35 & 69 \\
\hline Day 4 & 84 & 86 & 89 & 76 & 86 & 121 & 133 & 130 & 51 & 91 & 92 \\
\hline Day 5 & 102 & 109 & 116 & 92 & 129 & 144 & 169 & 127 & 98 & 112 & 106 \\
\hline Day 6 & 119 & 127 & 123 & 136 & 151 & 152 & 186 & 142 & 103 & 139 & 133 \\
\hline Day 7 & 136 & 162 & 147 & 142 & 155 & 178 & 216 & 159 & 142 & 158 & 164 \\
\hline Day 8 & 154 & 186 & 165 & 155 & 162 & 155 & 244 & 166 & 165 & 162 & 161 \\
\hline
\end{tabular}


apoptosis [26]. Pathophysiologically, any disruption in the platelets' life-cycle that reduces its blood count is termed a 'thrombocytopenia'. There are two mechanisms involved, either a reduction in platelet production or an elevation of platelet destruction [24].

A sudden drop in the platelet count below the lower limit results in overall poor therapeutic outcomes in ICU critically ill patients [5]. Both an acute or chronic decrease in the platelet count significantly increases the mortality and morbidity in ICU patients [7]. Akca et al. (2010) found that $25-55 \%$ of ICU patients developed thrombocytopenia in some stage of their hospitalization [27].

Sepsis was determined as the most common factor causing thrombocytopenia $[9,28,29]$.

Sepsis is an infectious syndrome etiologically based on altered immune responses to infections, including non-immunologic responses to multiple organ-functions and metabolic pathways [31]. The activation of innate immune responses in sepsis progression leads to a failure of systemic inflammatory conditions, and this process is triggered by pro-inflammatory mediators and innate-immune cell activation, in association with a high level of circulating cytokines [31-33]. As the consequence of sepsis or septic shock, systemic inflammatory responses are triggered by the endogenous immune-activating peptides termed alarmins [34] that are released by damaged tissues [35]. Again circulating platelets come in close contact with the ruptured vessels of septic patients and sequentially, followed by adherence, activation and aggregation, repair the damaged vessels [31].

In sepsis, because of their pivotal role in tissue homeostasis, directly or indirectly playing on different stages or factors of systemic inflammations, contributing to damaged vessels repairing and tissue regeneration, circulating platelet numbers are significantly reduced, leading to SAT [36-38].

Several mechanisms are responsible, alone or in combination, resulting in the development of a low platelet count during sepsis. Sepsis-associated bone marrow suppression causes reduced production of platelet triggered by pathogens, endotoxins and sepsis-induced inflammatory mediators $[31,39-41]$. The reduction in platelets' half-life, depletion in platelet production and destruction of circulatory platelets are linked to sepsisassociated intravascular coagulopathy and immune dysfunctions [31]. Sepsis coagulopathy, characterized by DIC, leucocytes-mediated platelet sequestration and vascular inflammatory mediators is the principal trigger of the mechanism of thrombocytopenia. Additionally, platelet plays a significant role in the pathogen clearance that also leads to thrombocytopenia $[31,38$, $39,41]$. An adequate number of circulatory platelets is utilized in sepsis-induced vascular integrity recovery to stop bleeding and cytokines-mediated haemophagocytosis of platelet ultimately, lead to thrombocytopenia $[31,42,43]$. Both the thrombotic microangiopathies (TMA) and the secondary TMA are a series of disorders including ITP as well as sepsis, and these are one of the primary cause of SAT [24]. Ghosh et al. (1999) used Direct Platelet Suspension Immunofluorescence Test (PSIFT) to identify the immunological mechanism behind SAT and found that 54\% patients with septicaemia represented antiplatelet antibodies and 67.5\% of those patients developed thrombocytopenia [44]. In patients with sepsis, circulating platelets not only participate in the body's haemostasis functions but are also directly involved in the management of infection [45].

Along with the innate immune cells, platelets form an immune-driven thrombus which regulates the development of neutrophil extracellular traps where bacteria and viruses are entrapped and killed [46]. Platelet can also directly kill bacteria by producing a microbicidal protein, also known as 'c' [47]. These activities result in the over-use of platelets beyond its haemostasis function [45] and a reduction in blood platelet count leading to the progression of thrombocytopenia [37, 38].

Eltrombopag is the second-line drug of choice for ITP management due to its good oral bioavailability with a peak serum concentration occurring after 2-6 hours of oral administration and a half-life of 21-32 hours [48]. Native thrombopoietin binds with the extracellular domain of the thrombopoietin receptor, whereas eltrombopag binds selectively to the transmembrane domain of the TPO-receptors [20]. In the in vitro and ex vivo studies, eltrombopag was not found with any effect on agonist-induced platelet aggregation or activation [49]. Eltrombopag stimulates megakaryocytopoiesis by using the Janus kinase/signal transducer and activator of complex transcription (JAK/STAT) signalling pathway in the cell $[20,49]$. The activities of sepsis mediated antiplatelet antibodies against the platelets are very similar to that of ITP-associated antiplatelet antibodies, and these are mostly the megakaryopoiesis inhibitor [17, $18,22,31]$. As a thrombopoietin mimetic, eltrombopag actively acts as a megakaryopoiesis stimulator where 
there is a sepsis-induced megakaryopoiesis inhibition, and in septic patients, an eltrombopag targeted receptor-mediated mechanical stimulation significantly increases the blood platelet count. 18, 20, 22, 31].

The current study data showed excellent results using the high dose of $100 \mathrm{mg}$ /day of eltrombopag for the SAT management in critically ill adult patients with $64.71 \%$ of the total thrombocytopenia events being resolved in eight days. No significant eltrombopag-induced mild to severe adverse reaction was found during the study time.

It is acknowledged that the small sample size, the short duration of the study and the location at a single ICU-setups were limitations factors in this study.

\section{CONCLUSION}

Sepsis-associated thrombocytopenia is a severe threat to septic patients in an ICU, leading to a high rate of mortality and morbidity. Eltrombopag is a new drug recommended for the treatment of ITP.

A high dose of eltrombopag reversed SAT in a significant number of critically ill adult patients in a short time frame with no evidence of adverse reaction being reported.

\section{ACKNOWLEDGEMENT}

All authors of this study are very grateful to all ICU doctors and authority of Square Hospital for permission to conduct this study and for their kind of cooperation and support.

\section{- FUNDING ACKNOWLEDGEMENTS}

This research received no specific grant from any funding agency in the public, commercial, or not-for-profit sectors.

\section{- CONFLICTS OF INTEREST}

No conflicts of interest to declare.

\section{REFERENCES}

1. Singer M, Deutschman CS, Seymour CW, et al. The Third International Consensus Definitions for Sepsis and Septic Shock (Sepsis-3). JAMA. 2016;315:801-10.

2. Kaukonen K, Bailey M, Suzuki S, et al. Mortality Related to
Severe Sepsis and Septic Shock Among Critically III Patients in Australia and New Zealand, 2000-2012. JAMA. 2014;311:130816.

3. Bone RC, Balk RA, Cerra FB, et al. American College of Chest Physicians/Society of Critical Care Medicine Consensus Conference: definitions for sepsis and organ failure and guidelines for the use of innovative therapies in sepsis. Crit Care Med. 1992;20:864-74.

4. Ventetuolo CE, Levy MM. Sepsis: a clinical update. Clin J Am Soc Nephrol. 2008;3:571-7.

5. Venkata C, Kashyap R, Farmer JC, et al. Thrombocytopenia in adult patients with sepsis: incidence, risk factors, and its association with clinical outcome. J Intensive Care. 2013;1:9.

6. Daly ME. Determinants of platelet count in humans. Haematol. 2011;96:10-3 .

7. Drews RE, Weinberger SE. Thrombocytopenic disorders in critically ill patients. Am J Respir Crit Care Med. 2000;162:34751.

8. Stephan F, Hollande J, Richard O, et al. Thrombocytopenia in a surgical ICU. Chest. 1999;115:1363-70.

9. Bonfiglio MF, Traeger SM, Kier KL, et al. Thrombocytopenia in intensive care patients: a comprehensive analysis of risk factors in 314 patients. Ann Pharmacother. 1995;29:835-42.

10. Cawley MJ, Wittbrodt ET, Boyce EG, et al. Potential risk factors associated with thrombocytopenia in a surgical intensive care unit. Pharmacotherapy. 1999;19:108-13.

11. Shalansky SJ, Verma AK, Levine $M$, et al. Risk markers for thrombocytopenia in critically ill patients: a prospective analysis. Pharmacotherapy. 2002;22:803-13.

12. Abraham E, Anzueto A, Gutierrez G, et al. Double-blind randomised controlled trial of monoclonal antibody to human tumour necrosis factor in treatment of septic shock. NORASEPT II study group. Lancet. 1998;351:929-33.

13. Cohen J, Carlet J. INTERSEPT: an international, multicenter, placebo-controlled trial of monoclonal antibody to human tumor necrosis factor-alpha in patients with sepsis. International sepsis trial study group. Crit Care Med. 1996;24:1431-40.

14. Munford RS, Suffredini AF. Sepsis, Severe sepsis, and septic shock. In Mandell GL, Bennett JE, Dolin R. (Eds.) Mandell, Douglas, and Bennett's Principles and Practice of Infectious Diseases. Seventh Edition. Philadelphia: Churchill Livingstone. 2010, pp. 987-1010.

15. Lambert MP, Gernsheimer TB. Clinical updates in adult immune thrombocytopenia. Blood. 2017;129:2829-35.

16. Gonzalez-Porras JR, Bastida JM. Eltrombopag in immune thrombocytopenia: efficacy review and update on drug safety. Ther Adv Drug Saf. 2018;9:263-85.

17. Kim TO, Despotovic J, Lambert MP. Eltrombopag for use in children with immune thrombocytopenia. Blood Adv. 2018;2:454-61.

18. Bussel JB, Cheng G, Saleh MN, et al. Eltrombopag for the 
Available online at: www.jccm.ro

treatment of chronic idiopathic thrombocytopenic purpura. N Engl J Med. 2007;357:2237-47.

19. Barrios NJ, Humbert JR, McNeil J. Treatment of acute idiopathic thrombocytopenic purpura with high-dose methylprednisolone and immunoglobulin. Acta Haematol. 1993;89:6-9.

20. Cheng G. Eltrombopag, a thrombopoietin- receptor agonist in the treatment of adult chronic immune thrombocytopenia: a review of the efficacy and safety profile. Ther Adv Hematol. 2012;3:155-64.

21. Saleh MN, Bussel JB, Cheng G, et al. Safety and efficacy of eltrombopag for treatment of chronic immune thrombocytopenia: results of the long-term, open-label EXTEND study. Blood. 2013;121:537-45.

22. McMillan $R$, Wang $L$, Tomer $A$, et al. Suppression of in vitro megakaryocyte production by antiplatelet autoantibodies from adult patients with chronic ITP. Blood. 2004;103:1364-9.

23. Nurden AT, Viallard JF, Nurden P. New-generation drugs that stimulate platelet production in chronic immune thrombocytopenic purpura. Lancet. 2009;373:1562-69.

24. Ali N, Auerbach HE. New-onset acute thrombocytopenia in hospitalized patients: pathophysiology and diagnostic approach. J Community Hosp Intern Med Perspect. 2017;7:157-67.

25. Kaushansky K. Thrombopoiesis. Semin Hematol. 2015;52:411.

26. Mason KD, Carpinelli MR, Fletcher JI, et al. Programmed anuclear cell death delimits platelet life span. Cell. 2007;128:1173-86.

27. Akca S, Haji-Michael P, de Mendonça A, et al. Time course of platelet counts in critically ill patients. Crit Care Med. 2002;30:753-6.

28. Cawley MJ, Wittbrodt ET, Boyce EG, et al. Potential risk factors associated with thrombocytopenia in a surgical intensive care unit. Pharmacotherapy. 1999;19:108-13.

29. Shalansky SJ, Verma AK, Levine $M$, et al. Risk markers for thrombocytopenia in critically ill patients: a prospective analysis. Pharmacotherapy. 2002;22:803-13.

30. Sharma B, Sharma M, Majumder M, et al. Thrombocytopenia in septic shock patients-a prospective observational study of incidence, risk factors and correlation with clinical outcome. Anaesth Intensive Care. 2007;35:874-80.

31. Dewitte A, Lepreux S, Villeneuve J, et al. Blood platelets and sepsis pathophysiology: A new therapeutic prospect in critical ill patients? Ann. Intensive Care. 2017;7:115.

32. Riedemann NC, Guo RF, Ward PA. The enigma of sepsis. J Clin Investig. 2003;112:460-7.

33. Aziz M, Jacob A, Yang WL, et al. Current trends in inflammatory and immunomodulatory mediators in sepsis. J Leukoc Biol.
The Journal of Critical Care Medicine 2019;5(4) • 129

2013;93:329-42.

34. Yang D, Han Z, Oppenheim JJ. Alarmins and immunity. Immunol Rev. 2017;280:41-56.

35. Xiao W, Mindrinos MN, Seok J, et al. A genomic storm in critically injured humans. J Exp Med. 2011;208:2581-90.

36. Nurden AT. Platelets, inflammation and tissue regeneration. Thromb Haemost. 2011;105:S13-33.

37. Garraud O, Hamzeh-Cognasse H, Pozzetto B, et al. Bench-tobedside review: platelets and active immune functions-new clues for immunopathology? Crit Care. 2013;17:236.

38. Nurden AT. The biology of the platelet with special reference to inflam-mation, wound healing and immunity. Front Biosci (Landmark Ed). 2018;23:726-51.

39. Thiolliere F, Serre-Sapin AF, Reignier J, et al. Epidemiology and outcome of thrombocytopenic patients in the intensive care unit: results of a prospective multicenter study. Intensive Care Med. 2013;39:1460-8.

40. Lim SY, Jeon EJ, Kim HJ, et al. The incidence, causes, and prognostic significance of new-onset thrombocytopenia in intensive care units: a prospective cohort study in a Korean hospital. J Korean Med Sci. 2012;27:1418-23.

41. Antier N, Quenot JP, Doise JM, et al. Mecha-nisms and etiologies of thrombocytopenia in the intensive care unit: impact of extensive investigations. Ann Intensive Care. 2014;4:24

42. Francois B, Trimoreau F, Vignon P, et al. Thrombocytopenia in the sepsis syndrome: role of hemophagocytosis and macrophage colony-stimulating factor. Am J Med. 1997;103:114-20.

43. Stephan F, Thioliere B, Verdy E, et al. Role of hemophagocytic histiocytosis in the etiology of thrombocytopenia in patients with sepsis syndrome or septic shock. Clin Infect Dis. 1997;25:1159-64.

44. Ghosh TK, Khan N, Malik A. Platelet auto-antibodies in septicaemic patients. Indian J Pathol Microbiol. 1999;42:31-5.

45. Graham SM, Liles WC. Platelets in sepsis: beyond hemostasis. Blood. 2016;127:2947-9.

46. Herter JM, Rossaint J, Zarbock A. Platelets in inflammation and immunity. J Thromb Haemost. 2014;12:1764-75.

47. Semple JW, Italiano JE Jr, Freedman J. Platelets and the immune continuum. Nat Rev Immunol. 2011;11:264-74.

48. GlaxoSmithKline. Promacta (eltrombopag tablets): US prescribing information. 2011. Available at http://us.gsk. com/products/assets/us_promacta.pdf [accessed 23 January 2019].

49. Erhardt J, Erickson-Miller CL, Tapley P. SB 497115-GR, a low molecular weight TPOR agonist, does not induce platelet activation or enhance agonist-induced platelet aggregation in vitro. Blood. 2004;104:59b. 